\title{
Blu-ray based optomagnetic aptasensor for detection of small molecules
}

Yang, Jaeyoung; Donolato, Marco; Pinto, Alessandro; Bosco, Filippo; Hwu, En-Te; Chen, Ching-Hsiu; Alstrøm, Tommy Sonne; Lee, Gwan-Hyoung; Schäfer, Thomas; Vavassori, Paolo

Total number of authors:

13

Published in:

Biosensors and Bioelectronics

Link to article, DOI:

10.1016/j.bios.2015.08.062

Publication date:

2016

Document Version

Peer reviewed version

Link back to DTU Orbit

Citation (APA):

Yang, J., Donolato, M., Pinto, A., Bosco, F., Hwu, E-T., Chen, C-H., Alstrøm, T. S., Lee, G-H., Schäfer, T., Vavassori, P., Boisen, A., Lin, Q., \& Hansen, M. F. (2016). Blu-ray based optomagnetic aptasensor for detection of small molecules. Biosensors and Bioelectronics, 75, 396-403. https://doi.org/10.1016/j.bios.2015.08.062

\section{General rights}

Copyright and moral rights for the publications made accessible in the public portal are retained by the authors and/or other copyright owners and it is a condition of accessing publications that users recognise and abide by the legal requirements associated with these rights.

- Users may download and print one copy of any publication from the public portal for the purpose of private study or research.

- You may not further distribute the material or use it for any profit-making activity or commercial gain

- You may freely distribute the URL identifying the publication in the public portal 


\section{Blu-ray based optomagnetic aptasensor for detection of small molecules}

Jaeyoung Yang ${ }^{\mathrm{a}, \mathrm{b}}$, Marco Donolato ${ }^{\mathrm{a}}$, Alessandro Pinto ${ }^{\mathrm{c}}$, Filippo Giacomo Bosco ${ }^{\mathrm{a}}$, En-Te Hwu $^{\mathrm{d}}$, Ching-Hsiu Chen ${ }^{\mathrm{d}}$, Tommy Sonne Alstrøm ${ }^{\mathrm{a}}$, Gwan-Hyoung Lee ${ }^{\mathrm{e}}$, Thomas Schäfer ${ }^{\mathrm{c}, \mathrm{f}}$, Paolo Vavassori $^{\mathrm{f}, \mathrm{g}}$, Anja Boisen ${ }^{\mathrm{a}}$, Qiao Lin ${ }^{\mathrm{b},{ }^{*}}$, Mikkel Fougt Hansen ${ }^{\mathrm{a},{ }^{*}}$

${ }^{a}$ Department of Micro- and Nanotechnology, Technical University of Denmark, DTU Nanotech, Building 345 East, DK-2800 Kongens Lyngby, Denmark

${ }^{b}$ Department of Mechanical Engineering, Columbia University, New York, NY 10027, United States

${ }^{c}$ POLYMAT, University of the Basque Country UPV/EHU, 20018 Donostia-San Sebastian, Spain

${ }^{d}$ Institute of Physics, Academia Sinica, Nankang, Taipei 11529, Taiwan

${ }^{e}$ Department of Materials Science and Engineering, Yonsei University, Seoul, 120-749, Republic of Korea

${ }^{f}$ IKERBASQUE, Basque Foundation for Science, 48011 Bilbao, Spain ${ }^{g}$ CIC nanoGUNE Consolider, 20018 Donostia-San Sebastián, Spain

*Corresponding authors. Fax: +4588 7762 (M.F. Hansen), +1 2128543304 (Q. Lin)

Email address: mikkel.hansen@nanotech.dtu.dk (M.F. Hansen), qlin@columbia.edu (Q. Lin) 


\begin{abstract}
This paper describes an aptamer-based optomagnetic biosensor for detection of a small molecule based on target binding-induced inhibition of magnetic nanoparticle (MNP) clustering. For the detection of a target small molecule, two mutually exclusive binding reactions (aptamer-target binding and aptamer-DNA linker hybridization) are designed. An aptamer specific to the target and a DNA linker complementary to a part of the aptamer sequence are immobilized onto separate MNPs. Hybridization of the DNA linker and the aptamer induces formation of MNP clusters. The target-to-aptamer binding on MNPs prior to the addition of linker-functionalized MNPs significantly hinders the hybridization reaction, thus reducing the degree of MNP clustering. The clustering state, which is thus related to the target concentration, is then quantitatively determined by an optomagnetic readout technique that provides the hydrodynamic size distribution of MNPs and their clusters. A commercial Blu-ray optical pickup unit is used for optical signal acquisition, which enables the establishment of a low-cost and miniaturized biosensing platform. Experimental results show that the degree of MNP clustering correlates well with the concentration of a target small molecule, adenosine triphosphate (ATP) in this work, in the range between $10 \mu \mathrm{M}$ and 10 mM. This successful proof-of-concept indicates that our optomagnetic aptasensor can be further developed as a low-cost biosensing platform for detection of small molecule biomarkers in an out-of-lab setting.
\end{abstract}

Keywords: Aptamer, Magnetic nanoparticles, Inhibition assay, Blu-ray, Optomagnetic readout 


\section{Introduction}

Magnetic nanoparticles (MNPs) have been widely used in biosensing for detection of various biomolecules such as nucleic acids (Göransson et al. 2010; Josephson et al. 2001; Liong et al. 2013; Zhang et al. 2013b), proteins (Aurich et al. 2006; Liang et al. 2011; Ranzoni et al. 2012; Zhang et al. 2013a), enzymes (Bamrungsap et al. 2011), cells (Herr et al. 2006) and small molecules (Sun et al. 2006; Wu et al. 2011; Yigit et al. 2007; Zhang et al. 2009). Due to the low magnetic susceptibility of biological compounds, the manipulation and detection of MNPs in biological media are not susceptible to interference from magnetic background, and this allows for the efficient and sensitive detection of biomolecules without the need for sample purification steps.

MNP-based detection of biomolecules has been applied to both surface-based (Bruls et al. 2009; Mani et al. 2009; Nash et al. 2012; Tekin et al. 2013; Wang et al. 2010; Wang and Gan 2009) and volume-based assays (Aurich et al. 2006; Bamrungsap et al. 2011; Chun et al. 2011; Göransson et al. 2010; Josephson et al. 2001; Liang et al. 2011; Liong et al. 2013; Ranzoni et al. 2012; Sun et al. 2006; Zhang et al. 2013a). The latter typically employs sandwich binding-induced clustering where a molecule of interest is bound to multiple receptors tethered onto MNP surfaces, resulting in the agglutination of MNPs. Accordingly, the addition of a target triggers clustering of MNPs, leading to changes in the size and the number of agglomerated particles with the analyte concentration, which is referred to as a clustering assay (Aurich et al. 2006; Baudry et al. 2006; Castañeda et al. 2007; Göransson et al. 2010; Josephson et al. 2001; Koh et al. 2009; Liang et al. 2011; Ling et al. 2010; Perez et al. 2002; Ranzoni et al. 2012; Zhang et al. 2013a). Such a volume-based MNP clustering assay is particularly appealing since it allows simple mix-and-read type measurements and reduced reaction time by use of external magnetic fields (Baudry et al. 2006).

Despite these advantages, the extensive use of MNP clustering assays has been impeded by the following limitations: 
First, the typical clustering assay is applicable only to a target molecule with multiple binding sites, but not feasible for small molecules due to their limited number of binding sites. Therefore, target binding-induced cluster disassembly strategies have been pursued using a competitive binding assay format (Sun et al. 2006) or structure-switching signaling aptamers (Wu et al. 2011; Yigit et al. 2007; Zhang et al. 2009). However, in the competition-based approach, the formation of target-functionalized MNPs via a covalent bond is not always viable because of lack of functional groups within such small molecules, and the binding affinities of aptamers in the structure-switching approach are frequently reduced by competition, blocking (Nutiu and Li 2003) and allosteric inhibition (Ricci et al. 2012) effects, thus lowering the detection sensitivity.

Second, current technologies to detect MNP clusters typically measure properties such as transverse relaxation time (Bamrungsap et al. 2011; Eberbeck et al. 2009; Kaittanis et al. 2011; Liang et al. 2011; Ling et al. 2010; Min et al. 2012; Perez et al. 2002), total sample magnetization (Michael et al. 2012), or light scattering (Chun et al. 2011; Michael et al. 2012). However, since these methodologies do not resolve signals with respect to the distribution of particle sizes, quantitative measurements of clusters in a mixture are readily interfered by background signals from a large amount of single MNPs or clusters of different sizes, thus lowering the accuracy and sensitivity.

Third, existing readout techniques require bulky and sophisticated electronic equipment such as nuclear magnetic resonance (NMR) spectroscopy, magnetometers equipped with accurate temperature control, or dynamic light scattering equipment, which in turn limit their applicability in an out-of-lab setting.

In this study, we develop an aptamer-based MNP clustering biosensor for detection of small molecules via particle size-selective measurements on a compact, simple, and inexpensive optomagnetic readout system. A conventional inhibition assay configuration is employed (Crowther 2000), where two types of MNPs are prepared, one tagged with an 
aptamer (apt-MNPs), a single stranded oligonucleotide receptor (Ellington and Szostak 1990), and the other with a sequence partially complementary to the aptamer (linker-MNPs), called a DNA linker. When a sample containing a target small molecule is incubated with apt-MNPs prior to the addition of linker-MNPs, the aptamer is folded into a three-dimensional structure stabilized by the target, referred to as adaptive binding (Da Costa et al. 2013; Gilbert et al. 2009; Hermann and Patel 2000). From a thermodynamic viewpoint, such a stabilization implies that in the presence of its cognate target, the overall free energy of the hybridization reaction between an aptamer and its complementary DNA sequence becomes less negative (Da Costa et al. 2013). Thus, the small target molecule behaves like an inhibitor, and the resulting affinity constant $K_{\text {eq }}$ of the aptamer for its complementary strand (DNA linker) is reduced and can be expressed as $K_{\mathrm{eq}}{ }^{\prime}=K_{\mathrm{eq}} /\left(1+[T] \times K_{\mathrm{a}}\right)$, where $[T]$ is the target concentration and $K_{\mathrm{a}}$ is the affinity constant for the aptamer-target binding. Consequently, since MNP clusters are formed by the aptamer-linker hybridization reaction, the degree of MNP clustering decreases with increasing target concentration. This target binding-induced particle clustering inhibition approach facilitates the detection of diverse small molecules by designing the linker strands to hybridize with known binding sequences of aptamers.

To determine the degree of MNP clustering, i.e., the cluster sizes and respective amounts, we implemented an optomagnetic readout system that collects transmitted light, which is modulated by rotating MNPs under the actuation of an external magnetic field (Donolato et al. 2015a). The optical signal is found to be closely related to the rotational dynamics of MNPs, which is linked to their hydrodynamic sizes. As a result, this optomagnetic measurement enables us to effectively detect optical signals with respect to cluster sizes, allowing an accurate and quantitative determination of the clustering state.

Furthermore, to simplify and miniaturize the readout platform, we utilize a Blu-ray optical pickup unit (OPU) that incorporates a laser diode and a photo detector costing less than $10 \$$ (Donolato et al. 2015b). By integrating such a compact and reliable optical element with a 
cuvette for simple sample loading (Fig. S1), we establish a robust and miniaturized optomagnetic readout system.

In this proof-of-concept study, adenosine triphosphate (ATP) is chosen as a target small molecule. ATP is an intracellular energy transporter that regulates cell metabolism and signaling pathways. ATP is also used as an important indicator of cell viability (Leist et al. 1997) and its intracellular concentration ranges from 0.1 to $3 \mathrm{mM}$ (Traut 1994). Thus, it is important to detect ATP in the range for clinical and biomedical research. Combining the aptamer-based inhibitive MNP clustering strategy with size-selective measurements on a lowcost and miniaturized platform, we demonstrate in this proof-of-concept study that our optomagnetic aptasensor can achieve the detection of ATP in the concentration range from 10 $\mu \mathrm{M}$ to $10 \mathrm{mM}$.

\section{Materials and methods}

\subsection{Chemicals and materials}

Streptavidin-coated cross-linked iron oxide (CLIO) nanoparticles with hydroxyethyl starch and nominal sizes of 80,250 and $500 \mathrm{~nm}$ were acquired from Micromod Partikeltechnologie GmbH (Rostock, Germany). Biotinylated ATP-specific aptamer (5'-ACC TGG GGG AGT ATT GCG GAG GAA GGT AAA AAA A-3'), DNA linker (5'-CAA TAC TCC CCC AGG TAA AAA AA-3') and control aptamer (5'-ACC TGG GGG AGT ATT AAA AAA AAA AAA AAA AAA A-3') were obtained from Integrated DNA Technologies, Inc. (Coralville, IA). Other chemical reagents including ATP, CTP, GTP, UTP, PBS buffer, $\mathrm{MgCl}_{2}$ were purchased from Sigma-Aldrich (St. Louis, MO).

\subsection{MNP surface functionalization}

MNPs with a nominal diameter of $80 \mathrm{~nm}$ were repeatedly washed with PBS buffer four times using centrifugation followed by magnetic separation and re-suspended at a concentration of $1 \mathrm{mg} / \mathrm{ml}$. The MNP suspension was then mixed with the aptamer or the 
linker solution $(2 \mu \mathrm{M})$ with a 1:1 volume ratio, and incubated for 30 min under shaking at room temperature. After the incubation, the oligonucleotide-functionalized MNPs were washed with PBS with $5 \mathrm{mM} \mathrm{MgCl}_{2}$ (PBSM, pH 7.4) three times, and re-suspended at a concentration of $1 \mathrm{mg} / \mathrm{ml}$ for subsequent experiments. The number of DNA oligos (aptamer or DNA linker) on a MNP surface is estimated to be about 18 based on the information provided by the MNP vendor.

\subsection{Experimental procedure}

Standard ATP samples at various concentrations were prepared in PBSM buffer solution (pH 7.4). An apt-MNP solution $(1 \mathrm{mg} / \mathrm{ml}, 6 \mu \mathrm{l})$ was first mixed with a $6 \mu 1$ of ATP sample (or with a PBSM buffer for blank experiments), and incubated for $1 \mathrm{~h}$ at room temperature. A linker-MNP solution $(1 \mathrm{mg} / \mathrm{ml}, 6 \mu \mathrm{l})$ was then added into the sample followed by placing the sample tube in the magnetic rack for 5 min to accumulate MNPs together. 15 min after removing the tube from the rack, $42 \mu 1$ of PBSM buffer was added to make the final volume as $60 \mu 1$, which fills up the measuring chamber of the optical cuvette.

\subsection{Optomagnetic measurement}

Once the sample was ready to measure with a volume of $60 \mu 1$, the cuvette was mounted on the sample loading stage located between the adjustable mirror and the Blu-ray OPU (Fig. S1). Each spectrum consisted of optomagnetic measurements for magnetic field excitation of amplitude $2 \mathrm{mT}$ at 26 logarithmically spaced frequencies $f$ from 1928 to $0.33 \mathrm{~Hz}$. The acquisition time for a single spectrum was about $2 \mathrm{~min}$. The Blu-ray OPU had a wavelength of $405 \mathrm{~nm}$. The light passed through the sample solution was reflected on the mirror and returned to the photo detector of the OPU through the sample. The collected optical signals were analyzed using lock-in technique in order to extract the second harmonic signal (Donolato et al. 2015b).

\section{Results and discussion}




\subsection{Design and principle of MNP clustering}

The principle of ATP detection using MNPs is illustrated in Fig. 1. A part of the linker sequence (CAA TAC TCC CCC AGG T) is designed to hybridize with the ATP-recognition site (ACC TGG GGG AGT ATT G) of the aptamer. Without ATP, apt-MNPs and linkerMNPs agglomerate through successive DNA hybridization processes between aptamers and linkers on respective MNP surfaces (Fig. 1a). However, in the presence of ATP, the aptamer changes its conformation to form a loop like structure that incorporates two ATP molecules (Lin and Patei 1997), thereby making the complementary aptamer sequence inaccessible to the linker sequence. Hence, when apt-MNPs are first incubated with an ATP sample followed by addition of linker-MNPs, aptamers preoccupied by ATP molecules do not hybridize with the linkers (Fig. 1b). Consequently, MNP clustering is less likely to occur, andthus a higher ATP concentration in a sample results in the formation of smaller and fewer MNP clusters.

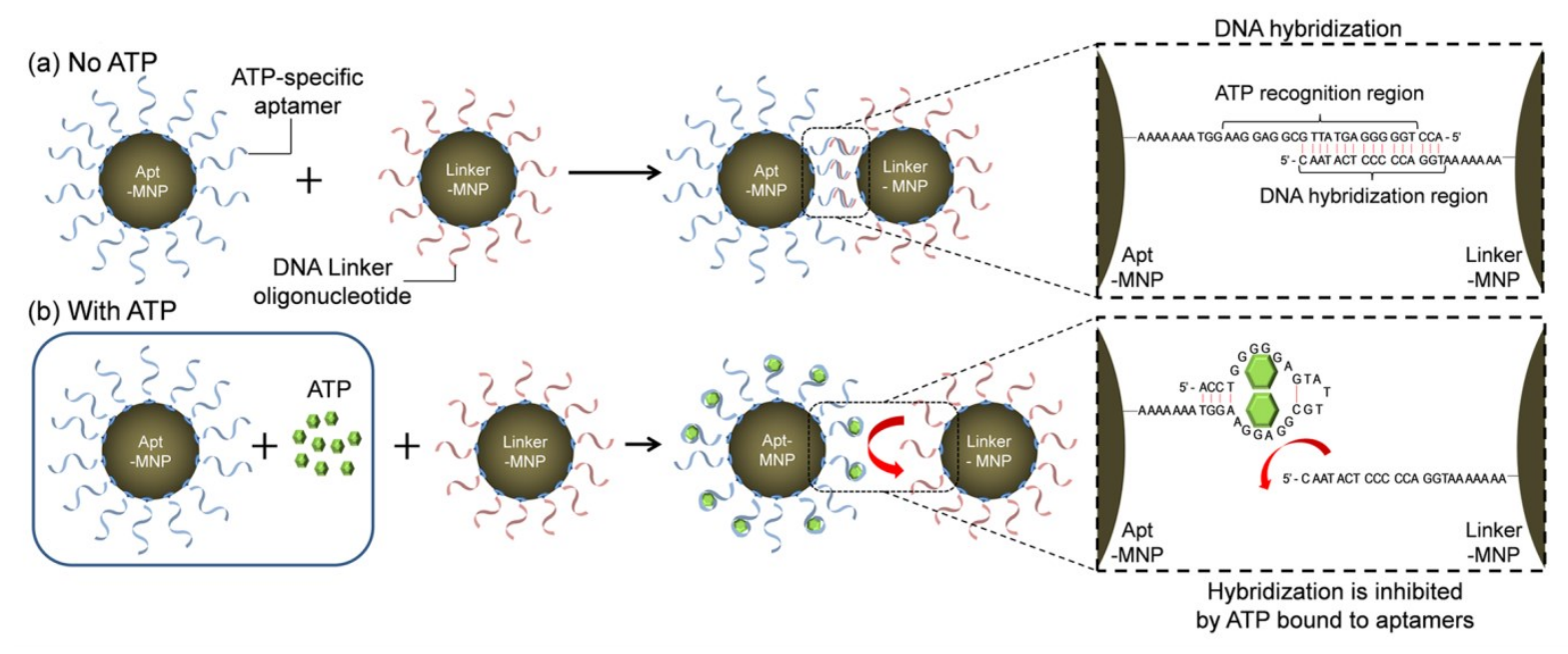

Fig. 1. Principle of ATP detection using oligonucleotide-conjugated MNPs. (a) When two types of MNPs (apt-MNPs and linker-MNPs) are mixed without ATP, hybridization between ATP-specific aptamers and linker DNA molecules induce agglutination of MNPs resulting in MNP clustering. (b) When apt-MNPs are first incubated with ATP samples followed by addition of linker-MNPs, aptamers pre-bound to ATP molecules do not hybridize with linkers and reduce the likelihood of cluster formation. 


\subsection{Image-based characterization of target binding-induced MNP clustering}

We first demonstrated the aptamer-based inhibition mechanism for ATP detection by taking images of the clustering state of MNP samples. Fig. 2 shows images of (a) a single type of MNPs (either apt- or linker-MNPs), (b) apt-MNPs mixed with an equal amount of linker-MNPs, and (c) apt-MNPs incubated with $10 \mathrm{mM}$ ATP followed by addition of an equal amount of linker-MNPs. The top and bottom images in Fig. 2 were obtained by fluorescence microscopy of fluorophore-labeled MNPs in suspension and scanning electron microscopy (SEM) of unlabeled MNPs after drying a sample droplet, respectively.

(a) Apt-MNPs only (or Linker-MNPs only)
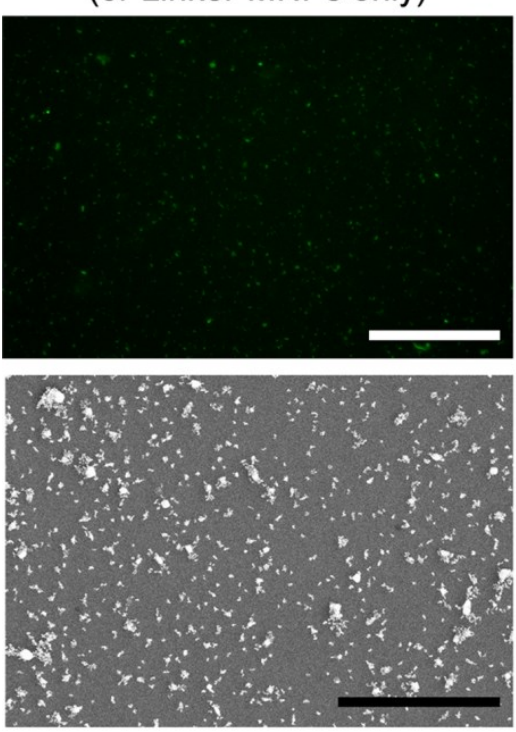

(b) Apt-MNPs + Linker MNPs
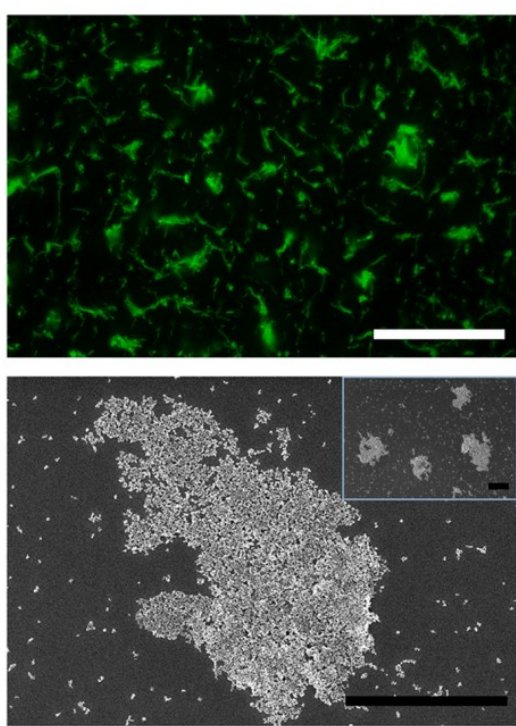

(c) [Apt-MNPs + $10 \mathrm{mM}$ ATP] + Linker MNPs
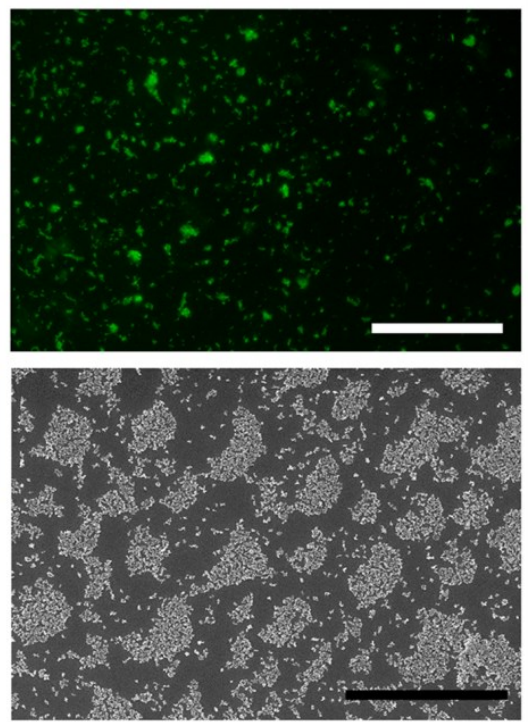

Fig. 2. Images of MNP samples prepared at different conditions. The top row shows fluorescence microscopic images acquired on suspensions and the bottom row shows SEM images acquired on dried spots of samples of (a) a single type of MNPs, (b) a 1:1 mixture of apt-MNPs and linker-MNPs, and (c) apt-MNPs incubated with $10 \mathrm{mM}$ APT followed by addition of linker-MNPs. The white and black scale bars indicate $20 \mu \mathrm{m}$ and $5 \mu \mathrm{m}$, respectively. 
The two techniques showed consistent results. For a single type of MNPs, the MNPs were uniformly dispersed with some small clusters $(<1 \mu \mathrm{m})$ formed by non-specific interaction between MNPs (Fig. 2a). For the mixture of apt-MNPs and linker-MNPs, large $(>10 \mu \mathrm{m})$ clusters were observed (Fig. 2b). When the apt-MNPs were incubated with a high concentration of ATP prior to addition of the linker-MNPs, only intermediate-sized $(<5 \mu \mathrm{m})$ clusters were observed (Fig. 2c). From the results, it is noted that MNP clustering is significantly suppressed in the presence of a high ATP concentration, confirming that ATP acts as an inhibitor and prevents aptamers from hybridizing with linkers. This inhibition mechanism is additionally verified using dynamic light scattering (Fig. S3).

\subsection{Characterization of the optomagnetic readout}

To quantitatively determine the clustering state of MNPs, we implemented an optomagnetic readout system, which can measure the size and the relative amount of MNPs/clusters (Fig. 3a). In the system, a sinusoidal uniaxial magnetic field of frequency $(f)$ is applied to an MNP suspension while laser light (405 nm wavelength) passes through it along the axis of the applied magnetic field and is reflected back to the Blu-ray unit by an adjustable mirror. The MNPs have a small remnant magnetic moment and are irregularly shaped (Ludwig et al. 2014). In the absence of an external magnetic field, the thermal energy is sufficiently large to prevent the formation of particle clusters due to magnetic interactions. However, in an applied magnetic field, MNPs/clusters align preferentially with the magnetic field due to their remnant magnetization, resulting in a modulation of the intensity of the transmitted light (Donolato et al. 2015a). Upon changing the sign of the magnetic field, the MNPs/clusters physically rotate to minimize their energy in the applied magnetic field. The time scale for these alternate processes is given by the Brownian relaxation dynamics of the MNPs/clusters. The rotation of the MNPs and MNP clusters during a cycle of the magnetic 
field causes the modulation of the transmitted light intensity at twice the excitation frequency. The transmitted light modulation vs. applied magnetic field frequency reflects the dynamic behavior of MNPs in suspension. In our previous work, it was demonstrated that the $2^{\text {nd }}$ harmonic component of analyzed optical signals is closely related to the Brownian relaxation dynamics of MNPs/clusters, which in turn gives information about the size of the particles in suspension (Donolato et al. 2015a; Donolato et al. 2015b). More details are given in Supplementary Information Section S1.

To demonstrate the optomagnetic detection principle, we first characterized the readout system using bare MNPs with various sizes. Spectra of the complex $2^{\text {nd }}$ harmonic photo detector signal $V_{2}=V_{2}^{\prime}+\mathrm{i} V_{2}^{\prime \prime}$ with in-phase component $V_{2}^{\prime}$ and out-of-phase component $V_{2}^{\prime \prime}$ were recorded at 26 logarithmically equidistant frequencies from $f=1928 \mathrm{~Hz}$ to $0.33 \mathrm{~Hz}$. Fig. 3b shows frequency spectra $\left(V_{2}^{\prime} v s . f\right)$ for three different particle sizes with nominal diameters of 80,250 and $500 \mathrm{~nm}$, which have peaks at distinct frequencies $\left(f_{\mathrm{p}}\right)$ of 170,10 and $1.9 \mathrm{~Hz}$, respectively. These peak frequencies are related to the Brownian relaxation frequency, i.e., the frequency with the maximum out-of-phase magnetic response with respect to the external magnetic field excitation (Donolato et al. 2015a; Donolato et al. 2015b). Consequently, the peak frequency varies depending on the hydrodynamic size of particles as larger particles tend to rotate out-of-phase at lower frequency compared to smaller nanoparticles. Interestingly, and consistent with our initial work (Donolato et al. 2015a), the peak for $250 \mathrm{~nm}$ MNPs has opposite sign compared to the other particle sizes, indicating that the dynamics of the particles modulate the $405 \mathrm{~nm}$ light with an opposite sign compared to the particles with nominal sizes of $80 \mathrm{~nm}$ and $500 \mathrm{~nm}$. We are currently characterizing and modeling this effect by varying the particle size and the light wavelength. We also demonstrated the use of the method to quantify relative amounts of respective MNP sizes in a mixture by examining peak ratios using MNPs with two different sizes, 80 and $250 \mathrm{~nm}$ (Fig. S2). 
(a)

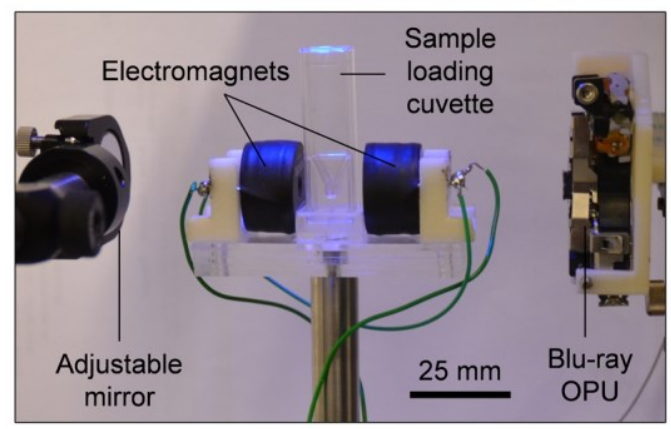

(b)

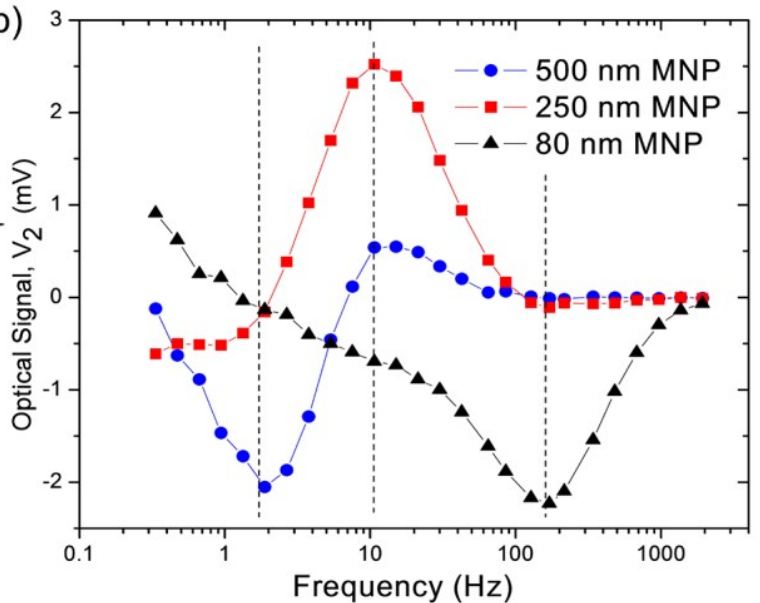

Fig. 3. (a) A photo of the Blu-ray OPU-based optomagnetic readout system. (b) $V_{2}^{\prime}$ spectra measured for MNP sizes of 500, 250 and $80 \mathrm{~nm}$ showing peaks (vertical dashed lines) at $f_{\mathrm{p}} \approx$ 1.9, 10 and $170 \mathrm{~Hz}$, respectively.

\subsection{Verification of the target binding-induced MNP clustering inhibition via optomagnetic} readout

We then utilized the optomagnetic readout system to test the aptamer-based inhibition mechanism for ATP detection using $80 \mathrm{~nm}$ MNPs. First, we obtained the characteristic spectrum from a blank sample of a 1:1 mixture of apt-MNPs and linker-MNPs without ATP (Fig. 4a, black squares). The spectrum exhibits three distinct peaks indicating that differentsized clusters are present in the suspension. Based on the results shown in Fig. 3b, we infer that the three peaks at $0.47,2.67$ and $170 \mathrm{~Hz}$ represent large clusters, intermediate-sized clusters and individual MNPs, respectively. Upon pre-incubation of apt-MNPs with $10 \mathrm{mM}$ ATP followed by the addition of linker-MNPs (Fig. 4a, red circles), the peak occurring at 0.47 $\mathrm{Hz}$ nearly disappeared, showing that the formation of large clusters is dramatically suppressed by high aptamer occupancy with ATP molecules. However, when only apt-MNPs are present in a sample, no appreciable difference is observed between the spectra with or without addition of ATP (Fig. 4b). Both spectra show the peak due to single MNPs with a shoulder appearing at around $10 \mathrm{~Hz}$. This shoulder is attributed to small clusters generated from nonspecific interactions between MNPs. The results are consistent with the images presented in 
Fig. 2 and also verify the cluster-size selectivity of the optomagnetic readout. The specificity of ATP detection in our aptasensor was confirmed by conducting aptamer- and analytecontrol experiments (Fig. S4). The results show that the inhibition of MNP clustering is due to specific binding of aptamers to ATP. More details are given in Supplementary Information Section S2.

(a) Apt-MNPs + Linker-MNPs

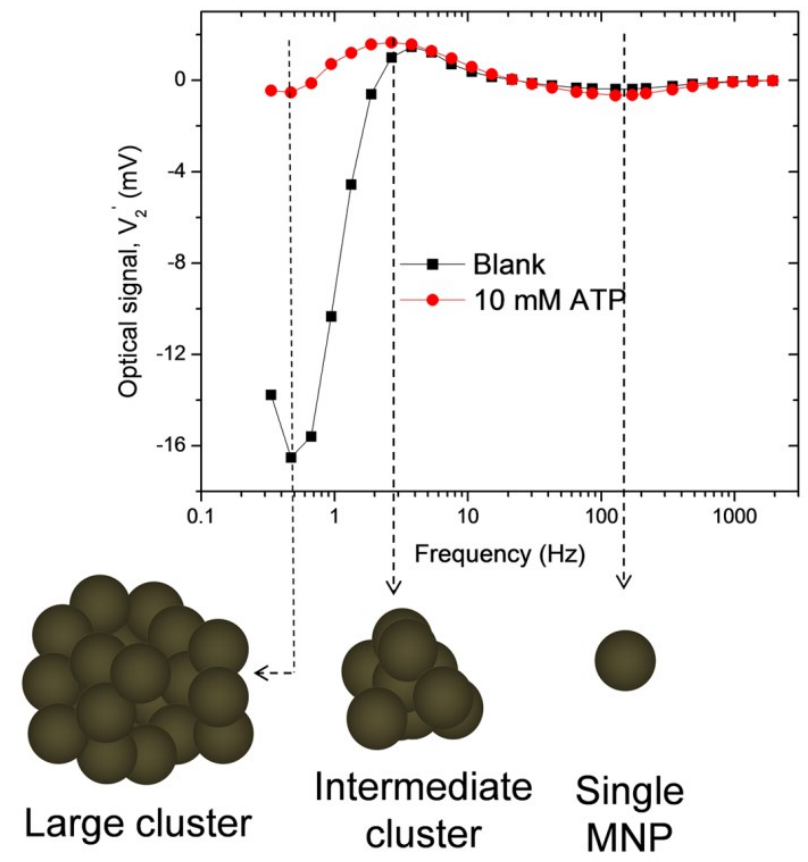

(b) Apt-MNPs only

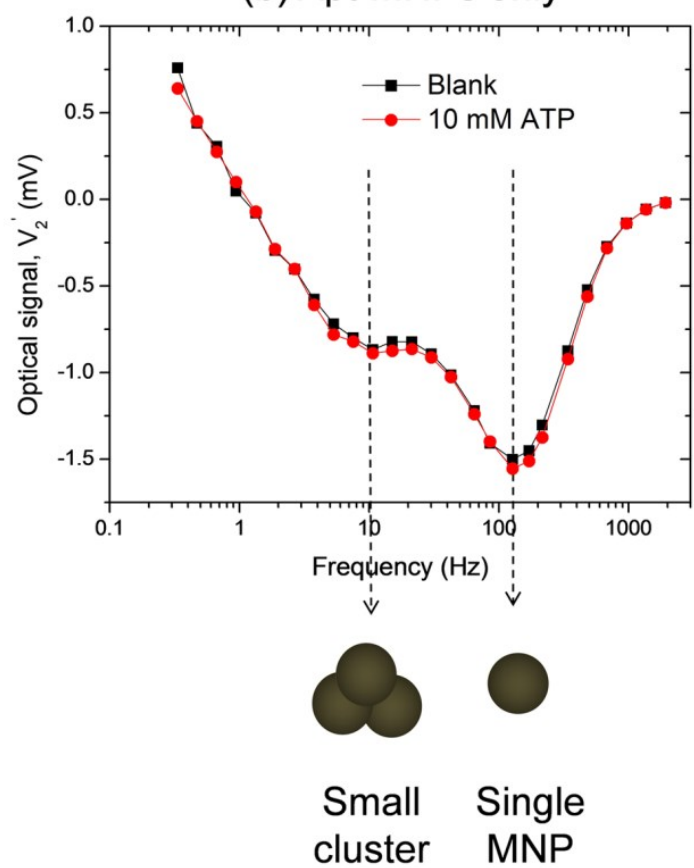

Fig. 4. Verification of inhibition-based ATP detection via optomagnetic readout. (a) $V_{2}^{\prime}$ spectra obtained for a mixture of apt-MNPs and linker-MNPs and for apt-MNPs incubated with $10 \mathrm{mM}$ ATP prior to mixing with linker-MNPs. (b) Spectra obtained for apt-MNPs with and without addition of $10 \mathrm{mM}$ ATP.

\subsection{ATP concentration-dependent signals}

Several experiments were carried out to evaluate the applicability of our optomagnetic aptasensor for ATP quantification in the range from 0 to $10000 \mu \mathrm{M}$. Apt-MNPs were incubated with suspensions of varying concentration of ATP followed by addition of linkerMNPs as described in the experimental section. For a more precise quantification of ATP 
concentration based on the signals, the spectra were divided into two regions (low- and highfrequency regions) containing the peaks representing the clustered (large and small clusters, see Fig. 4a) and single MNPs, respectively. We observed the following behavior for the frequency and magnitude of the peaks in the two regions $v s$. ATP concentration, $c_{\mathrm{ATP}}$ :

(1) Low- $f$ region in Fig. 5a: The magnitude of the negative peak at $0.47 \mathrm{~Hz}$ is observed to decrease with increasing $c_{\text {ATP }}$ between 0 and $500 \mu \mathrm{M}$, indicating that more ATP results in fewer large clusters in this concentration range. However, the effect on the dominating lowest frequency peak reaches saturation for $c_{\mathrm{ATP}} \geq 500 \mu \mathrm{M}$, implying that the formation of large clusters is significantly impeded by high aptamer-occupancies. Instead, we observe that the peak at intermediate frequency at $2.67 \mathrm{~Hz}$ decreases in magnitude for $c_{\mathrm{ATP}} \geq 500 \mu \mathrm{M}$. This clearly indicates that even the formation of intermediate-sized clusters is progressively hindered although at a lower rate. Further investigation and analysis of the shift of the dominating peak frequency is a topic for future work.

(2) High- $f$ region in Fig. 5a: The magnitude of the negative peak at $127 \mathrm{~Hz}$ attributed to

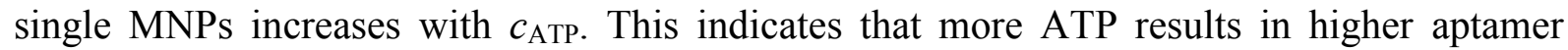
occupancies, hence impeding agglutination between apt-MNPs and linker-MPNs.

Based on the above qualitative observations, we conclude that the degree of MNP clustering sensitively depends on the ATP concentration and is reflected in the optomagnetic spectra. In order to derive a quantitative relationship between the measured optomagnetic spectra and the ATP concentration, we assumed that the intensity integral of the peaks in lowand high- $f$ regions is proportional to the amount of clustered and single MNPs, respectively. The validity of this assumption has been proven for magnetic susceptibility measurements and the results for mixtures of different particle sizes (Fig. S2) support its applicability also to our case. We define the relative fraction $R_{\text {free }}$ of the signal due to free MNPs as 


$$
R_{\text {free }}=\frac{\text { Free MNP signal }}{\text { Total MNP signal }}=\frac{\sum_{\text {high }-f}\left|V_{2}^{\prime}\right|}{\sum_{\text {all } f}\left|V_{2}^{\prime}\right|}
$$

where the numerator and denominator are total sums of magnitudes of peaks located in the high frequency region $(1928$ to $30 \mathrm{~Hz})$ and the whole region $(1928$ to $0.33 \mathrm{~Hz})$, respectively. Applying Equation (1) to the spectra in Fig. 5a, we found that $R_{\text {free }}$ monotonically increased with $c_{\text {ATP }}$ (Fig. $5 \mathrm{~b}$ ), confirming that the MNP clustering was reduced with increasing ATP concentrations in agreement with the aptamer-based inhibition mechanism. All experiments were carried out in triplicate. For blank samples, we obtained $R_{\text {free }}=0.037 \pm 0.005$, where the uncertainty indicates one standard deviation (s.d.). The horizontal red line in Fig. 5b shows the blank signal +3 s.d. corresponding to a limit of detection (LOD) of $74 \mu \mathrm{M}$. A dynamic range of at least 2 orders of magnitude was observed in the range between 0.1 and $10 \mathrm{mM}$, where $R_{\text {free }}$ linearly depends on $\log \left(c_{\text {ATP }}\right)$ according to $\mathrm{R}_{\text {free }}=0.072 \ln \left(c_{\mathrm{ATP}}\right)-0.2739$. This detection range covers the intracellular ATP concentration range (0.1-3 mM). Our detection capability is comparable to those of previously reported aptasensors for ATP detection (Chen et al. 2008; Song et al. 2012; Wang and Liu 2008; Wang et al. 2008; Zhou et al. 2012). Other aptasensors have shown ATP detection in the low nanomolar range (Lu et al. 2015; Wang et al. 2015; Xu et al. 2014) to demonstrate their capabilities for highly sensitive detection of small molecules. A further lowering of the detection limit may be achieved in our aptasensor by reducing the surface coverage of oligonucleotides (aptamer and/or DNA linker). It is also noted that the clustering is not fully inhibited even at mM ATP levels. This monotonic behavior in quantification is in accordance with the above qualitative observation on low- $f$ region in Fig. 5a that the inhibition effect grows with $c_{\text {ATP }}$ as it continues on clusters with smaller size. 

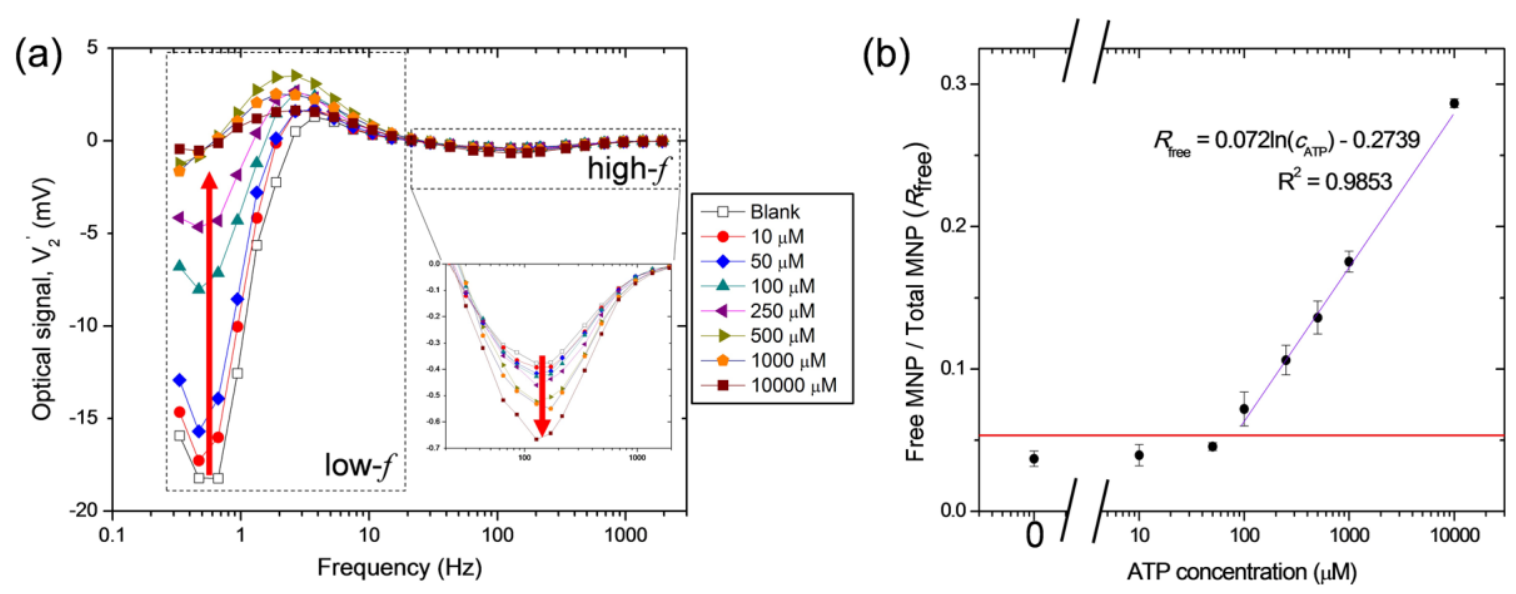

Fig. 5. (a) $V_{2}^{\prime}$ spectra obtained for the indicated ATP concentrations, where apt-MNPs (1 $\mathrm{mg} / \mathrm{ml}$ ) were incubated with the indicated concentrations of ATP followed by addition of linker-MNPs (1 mg/ml). The spectra are divided into two sections, low- and high- $f$ regions. The inset graph shows the magnified view of high- $f$ region Red arrows indicate changes of the spectra for increasing ATP concentration. (b) Relative signal fraction $R_{\text {free }}$ from free MNPs as a function of ATP concentration. Error bars show one standard deviation (s.d.) obtained from triplicate experiments. The red solid line shows the blank signal +3 s.d.

The error bars obtained from three replicates of the experiment are found to be large at low concentrations because large clusters tend to sediment out of the light path during a measurement in the current readout configuration (Fig. S5). This can be mitigated by using a vertical measurement configuration such as that presented in (Donolato et al. 2015b). The results presented in this proof-of-concept study using a model small molecule, ATP, demonstrate the potential of the optomagnetic aptasensor as a biosensing tool that can be widely applied to diverse small molecules by simply designing a DNA linker partially complementary to a specific known aptamer sequence. In addition, use of the compact Bluray OPU as an optical readout component allows the miniaturization of the setup and significantly reduces the total cost, showing its potential use as a point-of-care diagnostic tool that measures small molecule biomarker levels. 


\section{Conclusion}

We have developed an aptamer-based low-cost and simple optomagnetic biosensor for small molecule detection, and successfully demonstrated the detection of ATP in the biologically relevant range. A simple aptamer-based MNP clustering inhibition mechanism was employed for detection of ATP, wherein ATP binding to aptamers modulates the degree of MNP clustering. The clustering state was quantitatively measured with respect to the ATP concentration using an optomagnetic readout system, which collects the $2^{\text {nd }}$ harmonic optical transmission signal vs. frequency of an applied magnetic field. The acquired frequency spectra offer information regarding cluster sizes and relative amounts from peak frequencies and magnitudes, respectively. The accurate optomagnetic measurements of the size of MNPs/clusters enabled us to correlate optical signals to states of MNP clustering, from which ATP concentrations can be quantitatively and precisely estimated. We also demonstrated that the optomagnetic readout setup can be miniaturized by implementing compact and low-cost Blu-ray OPU. In conclusion, this was the first demonstration of a simple optomagnetic biosensing system for quantitative detection of small molecules on a low-cost Blu-ray optical readout platform. Our ongoing work includes a systematic study of the effect of the oligonucleotide coverage on the MNPs, which will allow us to lower the detection limit, as well as the application of the aptasensor to other small molecule biomarkers.

\section{Acknowledgements}

This work was financially supported by the ERC Advanced Grant No. 320535-HERMES, the Danish Strategic Research Council project MUSE and EU FP7 Grant No. 604448-NanoMag. J.Y. acknowledges the financial support from Raymond and Beverly Sackler Program at the Interfaces of Biophysical and Medical Sciences at Columbia University. M.D. gratefully acknowledges financial support from the Ørsted Postdoctoral Grant. P.V. acknowledges the 
Basque Government (Program No. PI2012-47) and the Spanish Ministry of Economy and Competitiveness (Project No. MAT2012-36844). G.H.L. acknowledges support from Basic Science Research Program (NRF-2014R1A1A1004632) through the National Research Foundation (NRF) funded by the Korean Government Ministry of Science, ICT and Future and in part by the Yonsei University Future-Leading Research Initiative of 2014. T.S. acknowledges support from the ERC Starting Grant No. 209842-MATRIX.

\section{Supplementary Information}

Supplementary data associated with this article can be found in the online version at

\section{References}

Aurich, K., Nagel, S., Glöckl, G., Weitschies, W., 2006. Determination of the MagnetoOptical Relaxation of Magnetic Nanoparticles as a Homogeneous Immunoassay. Anal. Chem. 79(2), 580-586.

Bamrungsap, S., Shukoor, M.I., Chen, T., Sefah, K., Tan, W., 2011. Detection of Lysozyme Magnetic Relaxation Switches Based on Aptamer-Functionalized Superparamagnetic Nanoparticles. Anal. Chem. 83(20), 7795-7799.

Baudry, J., Rouzeau, C., Goubault, C., Robic, C., Cohen-Tannoudji, L., Koenig, A., Bertrand, E., Bibette, J., 2006. Acceleration of the recognition rate between grafted ligands and receptors with magnetic forces. Proc. Natl. Acad. Sci. U.S.A. 103(44), 16076-16078.

Bruls, D.M., Evers, T.H., Kahlman, J.A.H., van Lankvelt, P.J.W., Ovsyanko, M., Pelssers, E.G.M., Schleipen, J.J.H.B., de Theije, F.K., Verschuren, C.A., van der Wijk, T., van Zon, J.B.A., Dittmer, W.U., Immink, A.H.J., Nieuwenhuis, J.H., Prins, M.W.J., 2009. Rapid integrated biosensor for multiplexed immunoassays based on actuated magnetic nanoparticles. Lab on a Chip 9(24), 3504-3510.

Castañeda, M.T., Merkoçi, A., Pumera, M., Alegret, S., 2007. Electrochemical genosensors for biomedical applications based on gold nanoparticles. Biosens. Bioelectron. 22(9-10), 1961-1967.

Chen, Z., Li, G., Zhang, L., Jiang, J., Li, Z., Peng, Z., Deng, L., 2008. A new method for the detection of ATP using a quantum-dot-tagged aptamer. Anal. Bioanal. Chem. 392(6), 1185 1188 .

Chun, C., Joo, J., Kwon, D., Kim, C.S., Cha, H.J., Chung, M.-S., Jeon, S., 2011. A facile and sensitive immunoassay for the detection of alpha-fetoprotein using gold-coated magnetic nanoparticle clusters and dynamic light scattering. Chem. Commun. 47(39), 11047-11049.

Crowther, J.R., 2000. The ELISA guidebook. Methods Mol. Biol. 149, Iii-iv, 1-413. 
Da Costa, J.B., Andreiev, A.I., Dieckmann, T., 2013. Thermodynamics and Kinetics of Adaptive Binding in the Malachite Green RNA Aptamer. Biochemistry 52(38), 6575-6583.

Donolato, M., Antunes, P., Bejhed, R.S., Zardán Gómez de la Torre, T., Østerberg, F.W., Strömberg, M., Nilsson, M., Strømme, M., Svedlindh, P., Hansen, M.F., Vavassori, P., 2015a. Novel Readout Method for Molecular Diagnostic Assays Based on Optical Measurements of Magnetic Nanobead Dynamics. Anal. Chem. 87(3), 1622-1629.

Donolato, M., Antunes, P., de la Torre, T.Z.G., Hwu, E.-T., Chen, C.-H., Burger, R., Rizzi, G., Bosco, F.G., Strømme, M., Boisen, A., Hansen, M.F., 2015b. Quantification of rolling circle amplified DNA using magnetic nanobeads and a Blu-ray optical pick-up unit. Biosens. Bioelectron. 67(0), 649-655.

Eberbeck, D., Wiekhorst, F., Steinhoff, U., Trahms, L., 2009. Quantification of biomolecule agglutination by magnetorelaxometry. Appl. Phys. Lett. 95(21), -.

Ellington, A.D., Szostak, J.W., 1990. In vitro selection of RNA molecules that bind specific ligands. Nature 346(6287), 818-822.

Gilbert, S.D., Reyes, F.E., Edwards, A.L., Batey, R.T., 2009. Adaptive ligand binding by the purine riboswitch in the recognition of guanine and adenine analogs. Structure 17(6), 857-868.

Göransson, J., Zardán Gómez De La Torre, T., Strömberg, M., Russell, C., Svedlindh, P., Strømme, M., Nilsson, M., 2010. Sensitive Detection of Bacterial DNA by Magnetic Nanoparticles. Anal. Chem. 82(22), 9138-9140.

Hermann, T., Patel, D.J., 2000. Adaptive recognition by nucleic acid aptamers. Science 287(5454), 820-825.

Herr, J.K., Smith, J.E., Medley, C.D., Shangguan, D., Tan, W., 2006. Aptamer-Conjugated Nanoparticles for Selective Collection and Detection of Cancer Cells. Anal. Chem. 78(9), 2918-2924.

Josephson, L., Perez, J.M., Weissleder, R., 2001. Magnetic Nanosensors for the Detection of Oligonucleotide Sequences. Angew. Chem. 113(17), 3304-3306.

Kaittanis, C., Santra, S., Santiesteban, O.J., Henderson, T.J., Perez, J.M., 2011. The Assembly State between Magnetic Nanosensors and Their Targets Orchestrates Their Magnetic Relaxation Response. J. Am. Chem. Soc. 133(10), 3668-3676.

Koh, I., Hong, R., Weissleder, R., Josephson, L., 2009. Nanoparticle-Target Interactions Parallel Antibody-Protein Interactions. Anal. Chem. 81(9), 3618-3622.

Leist, M., Single, B., Castoldi, A.F., Kühnle, S., Nicotera, P., 1997. Intracellular adenosine triphosphate (ATP) concentration: a switch in the decision between apoptosis and necrosis. The Journal of experimental medicine 185(8), 1481-1486.

Liang, G., Cai, S., Zhang, P., Peng, Y., Chen, H., Zhang, S., Kong, J., 2011. Magnetic relaxation switch and colorimetric detection of thrombin using aptamer-functionalized goldcoated iron oxide nanoparticles. Anal. Chim. Acta 689(2), 243-249. 
Lin, C.H., Patei, D.J., 1997. Structural basis of DNA folding and recognition in an AMPDNA aptamer complex: distinct architectures but common recognition motifs for DNA and RNA aptamers complexed to AMP. Chem. Biol. 4(11), 817-832.

Ling, Y., Vassiliou, C.C., Cima, M.J., 2010. Magnetic relaxation-based platform for multiplexed assays. Analyst 135(9), 2360-2364.

Liong, M., Hoang, A.N., Chung, J., Gural, N., Ford, C.B., Min, C., Shah, R.R., Ahmad, R., Fernandez-Suarez, M., Fortune, S.M., Toner, M., Lee, H., Weissleder, R., 2013. Magnetic barcode assay for genetic detection of pathogens. Nature communications 4, 1752.

Lu, L., Si, J.C., Gao, Z.F., Zhang, Y., Lei, J.L., Luo, H.Q., Li, N.B., 2015. Highly selective and sensitive electrochemical biosensor for ATP based on the dual strategy integrating the cofactor-dependent enzymatic ligation reaction with self-cleaving DNAzyme-amplified electrochemical detection. Biosens. Bioelectron. 63, 14-20.

Ludwig, F., Kazakova, O., Fernandez Barquin, L., Fornara, A., Trahms, L., Steinhoff, U., Svedlindh, P., Wetterskog, E., Pankhurst, Q., Southern, P., 2014. Magnetic, Structural, and Particle Size Analysis of Single-and Multi-Core Magnetic Nanoparticles. Magnetics, IEEE Transactions on 50(11), 1-4.

Mani, V., Chikkaveeraiah, B.V., Patel, V., Gutkind, J.S., Rusling, J.F., 2009. Ultrasensitive Immunosensor for Cancer Biomarker Proteins Using Gold Nanoparticle Film Electrodes and Multienzyme-Particle Amplification. ACS Nano 3(3), 585-594.

Michael, H., Thomas, H., Martin, Z., Christiane, B., Lothar, E., Jörg, B., John, E.W., Walter, R., Uwe, H., Marcel De, C., 2012. Magnetically triggered clustering of biotinylated iron oxide nanoparticles in the presence of streptavidinylated enzymes. Nanotechnology 23(35), 355707.

Min, C., Shao, H., Liong, M., Yoon, T.-J., Weissleder, R., Lee, H., 2012. Mechanism of Magnetic Relaxation Switching Sensing. ACS Nano 6(8), 6821-6828.

Nash, M.A., Waitumbi, J.N., Hoffman, A.S., Yager, P., Stayton, P.S., 2012. Multiplexed Enrichment and Detection of Malarial Biomarkers Using a Stimuli-Responsive Iron Oxide and Gold Nanoparticle Reagent System. ACS Nano 6(8), 6776-6785.

Nutiu, R., Li, Y., 2003. Structure-Switching Signaling Aptamers. Journal of the American Chemical Society 125(16), 4771-4778.

Özalp, V.C., Schäfer, T., 2011. Aptamer - Based Switchable Nanovalves for Stimuli Responsive Drug Delivery. Chemistry-A European Journal 17(36), 9893-9896.

Perez, J.M., Josephson, L., O'Loughlin, T., Hogemann, D., Weissleder, R., 2002. Magnetic relaxation switches capable of sensing molecular interactions. Nat. Biotechnol. 20(8), 816820.

Ranzoni, A., Sabatte, G., van Ijzendoorn, L.J., Prins, M.W.J., 2012. One-Step Homogeneous Magnetic Nanoparticle Immunoassay for Biomarker Detection Directly in Blood Plasma. ACS Nano 6(4), 3134-3141.

Ricci, F., Vallée-Bélisle, A., Porchetta, A., Plaxco, K.W., 2012. Rational Design of Allosteric Inhibitors and Activators Using the Population-Shift Model: In Vitro Validation and 
Application to an Artificial Biosensor. Journal of the American Chemical Society 134(37), 15177-15180.

Song, K., Kong, X., Liu, X., Zhang, Y., Zeng, Q., Tu, L., Shi, Z., Zhang, H., 2012. Aptamer optical biosensor without bio-breakage using upconversion nanoparticles as donors. Chem. Commun. 48(8), 1156-1158.

Sun, E.Y., Weissleder, R., Josephson, L., 2006. Continuous Analyte Sensing with Magnetic Nanoswitches. Small 2(10), 1144-1147.

Tekin, H.C., Cornaglia, M., Gijs, M.A.M., 2013. Attomolar protein detection using a magnetic bead surface coverage assay. Lab on a Chip 13(6), 1053-1059.

Traut, T., 1994. Physiological concentrations of purines and pyrimidines. Mol. Cell. Biochem. 140(1), 1-22.

Wang, J., Munir, A., Zhu, Z., Zhou, H.S., 2010. Magnetic Nanoparticle Enhanced Surface Plasmon Resonance Sensing and Its Application for the Ultrasensitive Detection of Magnetic Nanoparticle-Enriched Small Molecules. Anal. Chem. 82(16), 6782-6789.

Wang, K., Liao, J., Yang, X., Zhao, M., Chen, M., Yao, W., Tan, W., Lan, X., 2015. A labelfree aptasensor for highly sensitive detection of ATP and thrombin based on metal-enhanced PicoGreen fluorescence. Biosens. Bioelectron. 63, 172-177.

Wang, L., Gan, X.-X., 2009. Biomolecule-functionalized magnetic nanoparticles for flowthrough quartz crystal microbalance immunoassay of aflatoxin B1. Bioprocess Biosystems Eng. 32(1), 109-116.

Wang, Y., Liu, B., 2008. ATP detection using a label-free DNA aptamer and a cationic tetrahedralfluorene. Analyst 133(11), 1593-1598.

Wang, Y., Wang, Y., Liu, B., 2008. Fluorescent detection of ATP based on signaling DNA aptamer attached silica nanoparticles. Nanotechnology 19(41), 415605.

Wu, S., Duan, N., Wang, Z., Wang, H., 2011. Aptamer-functionalized magnetic nanoparticlebased bioassay for the detection of ochratoxin a using upconversion nanoparticles as labels. Analyst 136(11), 2306-2314.

Xu, Y., Xu, J., Xiang, Y., Yuan, R., Chai, Y., 2014. Target-induced structure switching of hairpin aptamers for label-free and sensitive fluorescent detection of ATP via exonucleasecatalyzed target recycling amplification. Biosens. Bioelectron. 51, 293-296.

Yigit, M.V., Mazumdar, D., Kim, H.-K., Lee, J.H., Odintsov, B., Lu, Y., 2007. Smart “Turnon" Magnetic Resonance Contrast Agents Based on Aptamer-Functionalized

Superparamagnetic Iron Oxide Nanoparticles. ChemBioChem 8(14), 1675-1678.

Zhang, S., Yan, Y., Bi, S., 2009. Design of Molecular Beacons as Signaling Probes for Adenosine Triphosphate Detection in Cancer Cells Based on Chemiluminescence Resonance Energy Transfer. Anal. Chem. 81(21), 8695-8701.

Zhang, X., Reeves, D.B., Perreard, I.M., Kett, W.C., Griswold, K.E., Gimi, B., Weaver, J.B., 2013a. Molecular sensing with magnetic nanoparticles using magnetic spectroscopy of nanoparticle Brownian motion. Biosens. Bioelectron. 50(0), 441-446. 
Zhang, Y., Pilapong, C., Guo, Y., Ling, Z., Cespedes, O., Quirke, P., Zhou, D., 2013 b. Sensitive, Simultaneous Quantitation of Two Unlabeled DNA Targets Using a Magnetic Nanoparticle-Enzyme Sandwich Assay. Anal. Chem. 85(19), 9238-9244.

Zhou, Z.-M., Yu, Y., Zhao, Y.-D., 2012. A new strategy for the detection of adenosine triphosphate by aptamer/quantum dot biosensor based on chemiluminescence resonance energy transfer. Analyst 137(18), 4262-4266. 